\author{
Rajeev Bhardwaj ${ }^{1 \star}$, Malay Sarkar ${ }^{2}$ and \\ Arvind Kandoria ${ }^{1}$ \\ ${ }^{1}$ Department of cardiology, Indira Gandhi medical \\ College, Shimla, India \\ ${ }^{2}$ Department of Pulmonary Medicine Indira Gandhi \\ medical College, Shimla, India
}

Dates: Received: 01 April, 2016; Accepted: 11 April, 2016; Published: 13 April, 2016

*Corresponding author: Dr. Rajeev Bhardwaj, Department of Cardiology, Indira Gandhi medical College, House no 24, Block 3, US Club, Shimla, 171001, E-mail: rajeevbhardwaj_dr@yahoo.com www.peertechz.com

ISSN: 2455-5452

Keywords: Hemoptysis; Embolization; Tuberculosis

\section{Research Article \\ Bronchial Artery Embolization for Moderate to Massive Hemoptysis}

\section{Abstract}

Purpose of study: Hemoptysis is a common emergency coming to the pulmonary medicine and general Medicine department. Massive hemoptysis has high mortality even after surgical treatment. Bronchial artery embolization is an effective alternative to surgery for controlling hemoptysis, with high success rate.

Material and methods: 74 consecutive patients coming to our hospital with moderate to severe hemoptysis were subjected to bronchial artery embolization (BAE). Femoral arterial puncture was the commonest approach. Some patients, where the culprit vessel was arising from subclavian artery, were approached from radial artery puncture. All patients were embolized with poly vinyl alcohol particles.

Results: Out of 74 patients, 54 were male and 20 were female. The mean age was $46.67 \pm 14.58$ yrs. Cause of hemoptysis was tuberculosis in 64 patients, bronchiectasis in two, aspergillosis in two and in six, the cause was not known. Total 192 vessels were embolized, 86 bronchial, 43 from subclavian, 53 intercostal and 20 internal mammary.

Within one year, recurrence occurred in 13 patients three of whom died. In 9 patients, the bleeding was controlled with repeat BAE.

Conclusion: Commonest cause of hemoptysis was pulmonary tuberculosis. BAE had initial success of $100 \%$. Recurrence occurred in $13(17.56 \%)$ patients. Repeat BAE was successful in majority of these. 3 patients died of recurrent hemoptysis
\end{abstract}

\section{Introduction}

Massive hemoptysis is a serious condition requiring urgent treatment. Despite advances in surgical and medical management of hemoptysis, it is still a major cause of morbidity and mortality. People have proposed various definitions for massive hemoptysis, the most acceptable being expectoration of $240 \mathrm{ml}$ blood in 24 hours [1]. In $90 \%$ of cases, significant hemoptysis occurs from bronchial artery [2]. Because the surgical mortality rate in emergency situations is around $40 \%$, bronchial artery embolization (BAE) has emerged as good alternative for management of hemoptysis [3-5].

\section{Materials and Methods}

Patients admitted with moderate to severe hemoptysis in pulmonary medicine or general medicine department were the subjects of study. The patients were subjected to routine blood examination, X ray of chest, and CT scan of chest (Except in emergent situation). If required, bronchoscopy was done to localize the side of bleeding and for diagnostic purposes. Informed consent was taken from all patients. Commonest approach was from right femoral artery puncture. Seven French sheath was inserted in the femoral artery with the help of Seldienger technique. According to the protocol, bronchial arteries, intercostal arteries, subclavial arteries and its branches including the internal mammary arteries were selectively hooked with a catheter and contrast was injected into these arteries to see for the signs of bleeding. Initial attempt was made to see for bleeding source on the side suggested by X-ray or CT scan or bronchoscopy. Before the completion of procedure, vessels of other side were screened. If no bleeding vessel was found after screening on both sides, pulmonary arteriography was done with pig tail catheter to see for the source of bleeding from pulmonary artery. An artery was considered abnormal/ potential bleeder, if extravasation of contrast, aneurysm, increased caliber with tortuosity, vascular tufts, arterio-arterial or arterio-venous fistulas were seen. Once diseased artery was identified, it was selectively cannulated with a catheter. Most commonly used catheters were right Judikin, Cobra I, and Simmon I. The vessels were hooked deeply with the catheter, with the support of .018 inch guide wire. The vessel was then embolized with poly vinyl alcohol (PVA) particles mixed with contrast agent. The particles were injected till vessel was occluded or reflux of contrast was seen. If a vessel could not be hooked deeply, a micro-catheter was passed through the catheter and PVA particles were injected through micro-catheter. Size of particles used was 500-700 microns. If it was difficult to cannulate the branches of subclavian artery selectively, due to fibrosis, the artery was approached from radial artery puncture. Criteria used for successful embolization was that all diseased arteries were embolized. If spinal artery was seen on cannulation of a bronchial artery, the embolization was done distal to its origin to avoid inadvertent embolization of spinal artery.

Patients were watched for recurrence of hemoptysis in the hospital for 3-4 days. Follow up was done after 2 weeks, then monthly for six months and then after one year. The patients were asked to report back in case of recurrence of bleeding.

\section{Results}

Total 74 patients were subjected to embolization. 74 were male and 20 were female. Mean age was $46.67 \pm 14.58$ yrs. Majority of 


\begin{tabular}{|l|l|l|}
\hline Table 1: Patient characteristics and etiology. \\
\hline Total patients & & 74 \\
\hline Male & & $54((72.9 \%)$ \\
\hline Female & $20(27.01 \%)$ \\
\hline Mean age & $46.67 \pm 14.58$ yrs. \\
\hline Dyspnea & $60(81.08 \%)$ \\
\hline Systolic BP $<100 \mathrm{mmHg}$ & $40(54.05 \%)$ \\
\hline Duration of hemoptysis & & \\
\hline$<7$ days & & $40(54.05 \%)$ \\
\hline $7-14$ days & $26(35.13 \%)$ \\
\hline $14-30 d a y s$ & $4(5.4 \%)$ \\
\hline$>1$ month & & $4(5.4 \%)$ \\
\hline Etiology & & $64(86.48 \%)$ \\
\hline Tuberculosis & & \\
\hline Treated & 57 & \\
\hline Active & 7 & $02(2.7 \%)$ \\
\hline Bronchiectasis & & $02(2.7 \%)$ \\
\hline As per gillosis & & $06(8.1 \%)$ \\
\hline Unknown & & \\
\hline & & \\
\hline
\end{tabular}

Table 2: Results of Embolization.

\begin{tabular}{|l|l|l|}
\hline Vessels embolized & & 192 \\
\hline No culprit vessel & & 4 patients (5.4\%) \\
\hline No of vessels /patient & & 2.74 \\
\hline Bronchial & & $53(75.7 \%)$ \\
\hline Only bronchial & & 19 patients (27.4\%) \\
\hline Only systemic & 21 patients (30\%) \\
\hline Bronchial with systemic & 34 patients (48.57\%) \\
\hline No of Bronchial vessels embolized & & $86(44.79 \%)$ \\
\hline Non Bronchial vessels embolized & & $118(61.45 \%)$ \\
\hline Intercostals & $53(27.6 \%)$ & \\
\hline Internal mammary & $20(10.4 \%)$ & \\
\hline Other arteries form subclavian & $43(22.39 \%)$ & \\
\hline Recurrence (no of patients) & & $13(18.57 \%)$ \\
\hline Successful embolization & $9(69.2 \%)$ & \\
\hline Death & $3((23.07 \%)$ & \\
\hline No culprit vessel & $1(7.6 \%)$ & \\
\hline Major complication & & Nil \\
\hline
\end{tabular}

patients had duration of hemoptysis of less two weeks. Around $50 \%$ patients had systolic BP less than $100 \mathrm{~mm} \mathrm{Hg}$. All patients had hemoglobin less than $10 \mathrm{gm} \%, 20$ patients had hemoglobin less than 7 gm. \%. Blood transfusion was required pre procedure in 52 patients. 8 patients required blood transfusion during the embolization. Table 1 shows the patient characteristics. It can be seen that the commonest cause of hemoptysis was tuberculosis, seen in 64 patients. 57 of these had been treated for tuberculosis, with mean duration after completion of anti-tubercular therapy (ATT) to bleeding being $10.53 \pm$ 5.82yrs. 7 patients had active tuberculosis, 3 of these had multi drug resistant tuberculosis. Two patients had bronchiectasis and 2 had as per gliosis. In 6 patients, the cause of hemoptysis was not clear. In three of these, culprit vessel could not be found and so embolization was not done. Table 2 shows the vessels embolized. In all, 192 vessels were embolized, commonest being 86 bronchial arteries (44.8\%), followed by 63 branches from subclavian artery(32.8\%) including 20 internal mammary (10.4\%). 53 (26.6\%) intercostals arteries were also embolized. Recurrence of bleeding occurred in 13 patients, 3 of whom died of massive hemoptysis. In the remaining 10 patients, BAE was attempted again. In one of these, no bleeding vessel was found. In 9 patients, 16 vessels were again embolized. Two patients required embolization third time.

\section{Discussion}

Massive hemoptysis commonly occurs in patients with history of chronic inflammatory disease. In western countries, the nontubercular causes such as malignancy and cystic fibrosis are most frequent [6]. In developing countries including India, pulmonary tuberculosis account for majority of the cases. In $90 \%$ cases of massive hemoptysis, bronchial circulation is responsible and so can be treated with bronchial artery embolization. In around 5\% cases, the culprit vessels are other systemic

Vessels or vessels arising directly from aorta. In around 5\% cases, the pulmonary arteries are responsible [2].

The pulmonary arteries and veins are a relatively low-pressure system, arising from the right heart, which takes part in gas exchange and makes the greatest contribution to pulmonary blood supply. The bronchial arteries, from a high-pressure system, provide nourishment to mediastinal, hilar, and pulmonary structural tissues and account for around $1 \%$ of pulmonary blood supply [7]. Bronchial vessels have also been shown to anastomose with branches of the subclavian, internal mammary, pericardiophrenic, esophageal, intercostal, and other systemic arteries [8]. These connections are important in both

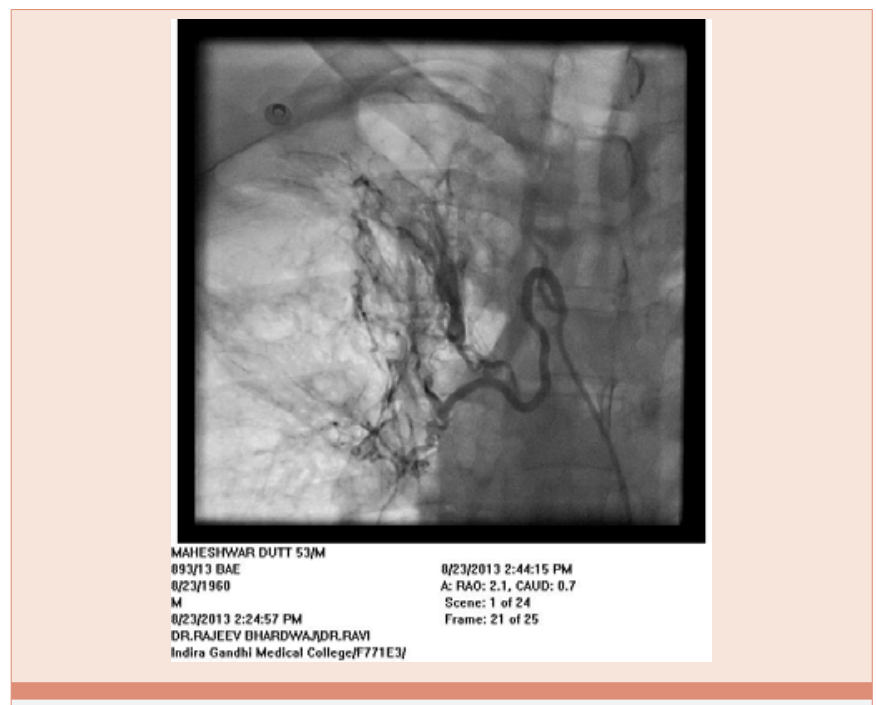

Figure 1: Embolization of right bronchial artery. 


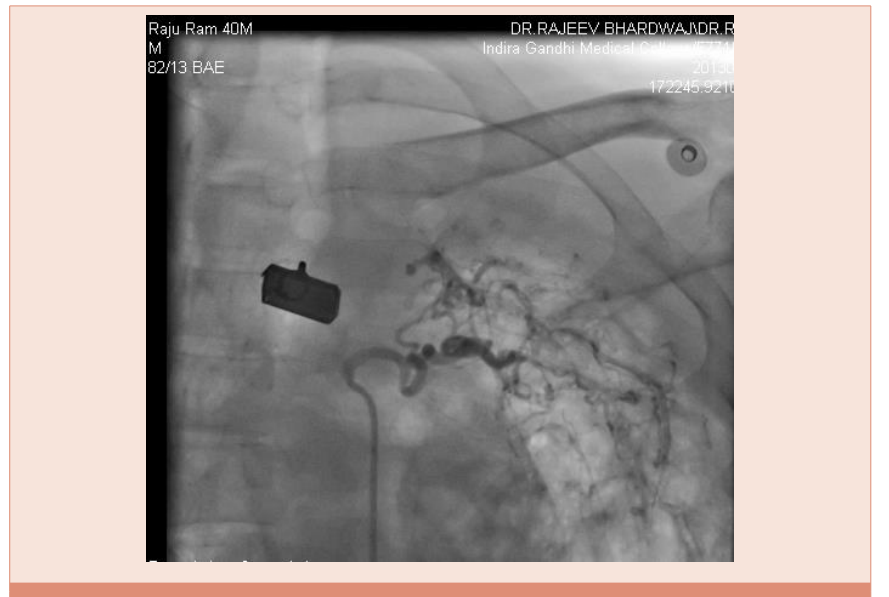

Figure 2: Source of bleeding from left bronchial artery.

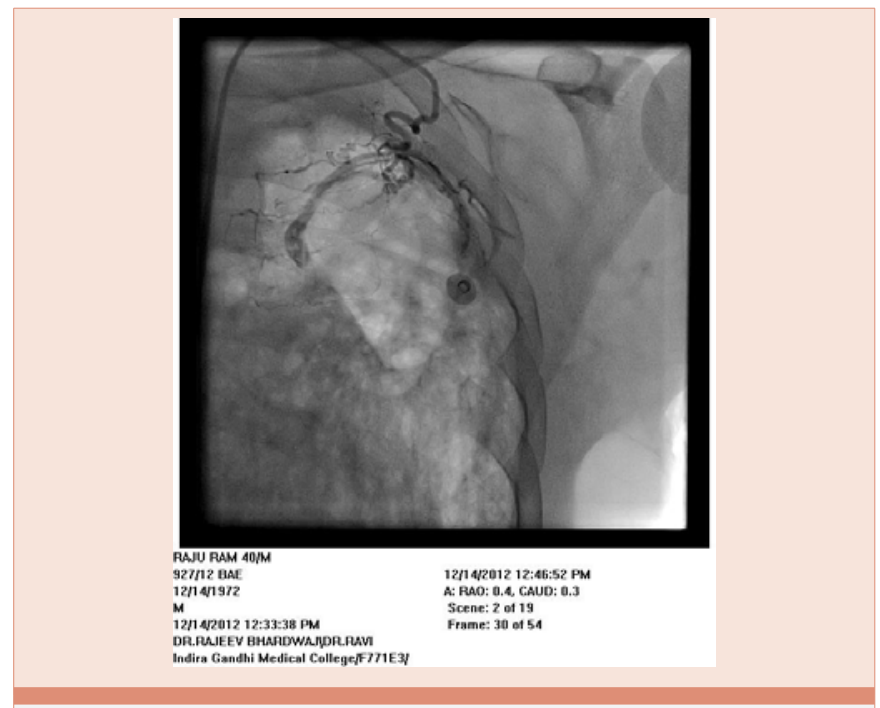

Figure 3: : Extravasation of contrast from a branch of left subclavian artery.

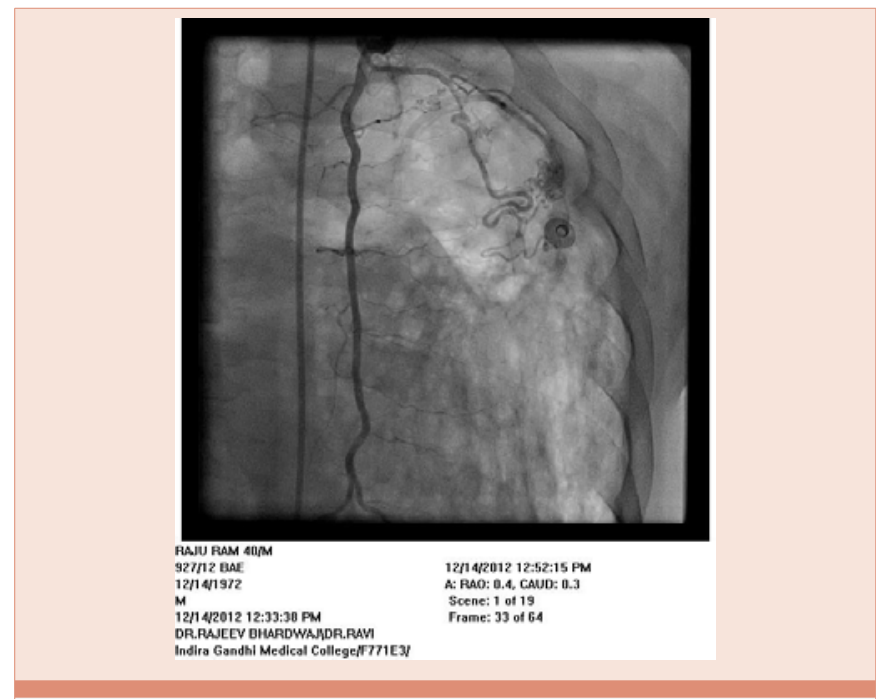

Figure 4: Culprit vessel is a branch of left internal mammary artery.

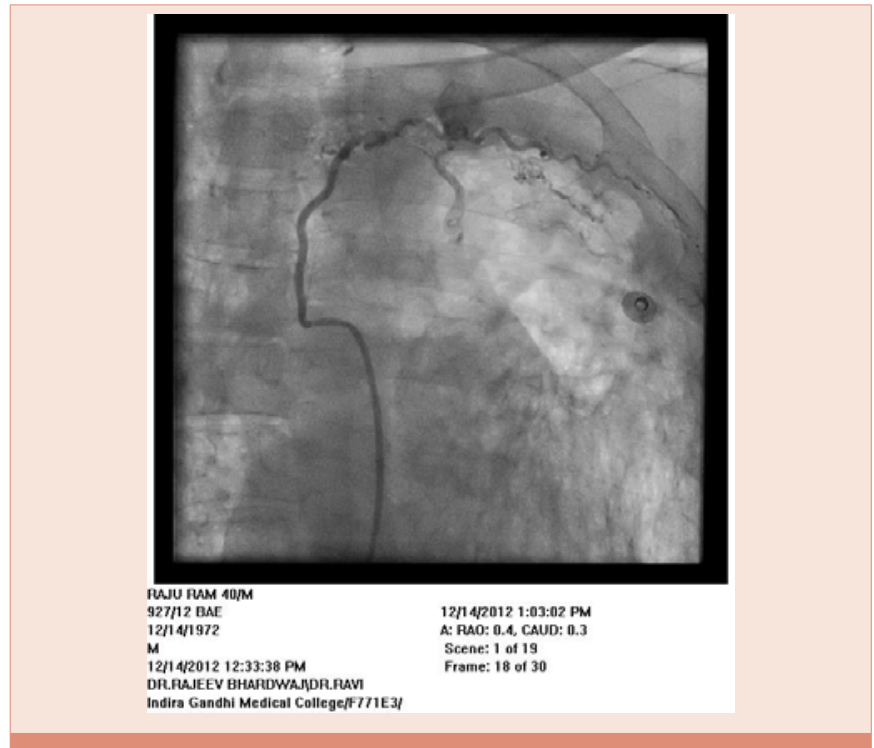

Figure 5: Extravasation of contrast from left upper intercostal artery.

understanding the pathophysiology of hemoptysis and the potential risks of embolotherapy. The first report of BAE to control hemoptysis was published by Re'my et al., in 1974 [9]. In 1976, this was followed by Wholey et al., [10], report of four cases and then the first large series by Re'my et al., in 1977 [4,11].

Our study shows that in around $86 \%$ cases, the cause of hemoptysis requiring embolization is tuberculosis. Most of these cases had completed the course of ATT long ago. Mean duration from ATT to hemoptysis in these patients was more than 10 years. Swansen et al., reported 54 cases of BAE. The etiology of the hemoptysis included bronchiectasis in nine patients (17\%), undefined in eight (15\%), malignancy in seven (13\%), pulmonary hypertension secondary to congenital heart disease in seven (13\%), mycetoma in seven (13\%), arteriovenous fistula in three (5\%), radiation fibrosis in three (5\%), tuberculosis in two $(3 \%)$, primary pulmonary hypertension in two (3\%), fibrosing mediastinitis in one (2\%), bronchial artery aneurysm in one (2\%), bronchial-to-pulmonary artery fistula in one (2\%), bronchial artery varices in one (2\%), a right middle-lobe bronchusto-bronchial artery fistula in one (2\%), and an aorta-to-bronchial fistula at the site of a previous aneurysm repair in one (2\%) [11].

We found that most of the cases required embolization of multiple vessels. In around half of the patients, both bronchial and systemic vessels required embolization. In around one fourth of patients, only bronchial and in another one fourth, only systemic vessels were the source of bleeding. In contrast to other studies, out of 192 vessels embolized, around 37\% vessels were arising from subclavian arteries, (including 10\% internal mammary arteries) and in $27 \%$ intercoastal arteries were the source of bleeding. Swansen et al., embolized the following arteries: right bronchial artery (34), left bronchial artery (11); combined right and left bronchial trunk (9); intercostal arteries (45); right internal mammary artery (8); left internal mammary artery (8); inferior phrenic artery (10); lateral thoracic arteries (3); thyrocervical trunk (2); and a left gastric artery (1) [11]. The complications of BAE in this study included subintimal 
dissection in two patients (4\%), arterial perforation by a guide wire in one patient (2\%), and reflux of embolic material into the aorta without adverse sequelae in one patient (2\%). Pleuritic chest pain post procedure occurred in two patients (4\%), shoulder pain post procedure in one patient $(2 \%)$, and transient dysphagia in one patient (2\%). One patient developed a groin hematoma at the puncture site. (Figures 1-5)

Immediate control of hemoptysis was achieved in all patients. Cremaschi et al., evaluated 209 patients who had been embolized for hemoptysis and noted that immediate control was achieved after BAE in 205 (98\%) [12]. Rabkin et al., evaluated 306 patients and found that BAE controlled acute bleeding in 278 (91\%) [13].

Recurrence of hemoptysis was quite common, occurring in around $18 \%$ patients, though most of these cases responded to repeat procedure. All 3 deaths occurred with recurrent massive hemoptysis. Three patients that we embolized had recurrence of hemoptysis after surgical lobectomy.

In the series by Mal et al. [14], seven patients had recurrent hemoptysis within 1 month of undergoing embolotherapy (16\%). In four of these patients, the bleeding developed within 10 days, and a second embolization procedure was performed. In these patients, an additional bronchial or nonbronchial collateral artery was embolized resulting in the successful control of hemoptysis. None of their patients had a recurrence of hemoptysis secondary to recanalization. They further analyzed their outcomes by the type of embolization material that was used. In six patients who developed recurrent hemoptysis within 1 month post procedure, the embolization materials used included the following: gelatin sponge (two patients); microspheres (two patients); polyvinyl alcohol (one patient); and gelatin sponge plus bucrylate (one patient).

The most feared complication of bronchial artery embolization is spinal cord stroke. This risk exists because the anterior spinal artery or artery of Adamkiewicz can originate from a bronchial artery in up to $5 \%$ of patients, more often on the right side [14]. This complication can be avoided if artery is carefully looked for, as it is seen as a small longitudinal vessel on the ventral surface of spinal cord and it takes characteristic hair pin turn after origin from aorta. If it is seen arising from bronchial artery, the catheter should be placed far beyond its origin or micro catheter should be used to embolize distal to its origin. Also if we use large particles ( $>500$ micrgrams), we can avoid this complication, since the size of spinal artery is less than 350 micron.

\section{Conclusion}

Commonest cause of hemoptysis requiring intervention in our set up is treated pulmonary tuberculos. BAE is lifesaving procedure. Most of the patients require embolization of multiple vessels. Both systemic and bronchial vessels need to be looked for, to avoid failure of therapy. Recurrence rate of bleeding is high but fortunately respond to repeat embolization.

\section{References}

1. Brinson GM1, Noone PG, Mauro MA, Knowles MR, Yankaskas JR, et al. (1998) Bronchial artery embolization for the treatment of hemoptysis in patients with cystic fibrosis. Am J Respir Crit Care Med 157: 1951-1958.

2. Yoon W, Kim J, Kim Y, Chung T, Kang H (2002) Bronchial and nonbronchial systemic artery embolization for life-threatening hemoptysis: a comprehensive review. Radiographics 22: 1395-1409.

3. Magilligan DJ Jr, Ravipati S, Zayat P, Shetty PC, Bower G, et al. (1981) Massive hemoptysis: control by transcatheter bronchial artery embolization. Ann Thorac Surg 32: 392-400.

4. Re'my J, Arnaud A, Fardou H, Giraud R, Voisin C (1977) Treatment of hemoptysis by embolization of bronchial arteries. Radiology 122: 33-37.

5. White RI Jr (1999) Bronchial artery embolotherapy for control of acute hemoptysis: analysis of outcome. Chest 115: 912-915.

6. Hirshberg B, Biran I, Glazer M, Kramer MR (1997) Hemoptysis: etiology, evaluation, and outcome in a tertiary referral hospital. Chest 112: 440-444.

7. Thompson AB, Teschler H, Rennard SI (1992) Pathogenesis, evaluation, and therapy for massive hemoptysis. Clin Chest Med 13: 69-82.

8. Liebow AA, Hales MR, Lindskog GE (1949) Enlargement of the bronchial arteries, and their anastomoses with the pulmonary arteries in bronchiectasis. Am J Pathol 25: 211-231.

9. Re'my J, Voisin C, Dupuis C (1974) Traitement des hemoptysies par embolisation de la circulation systemique. Ann Radiol (Paris) 17: 5-16.

10. Wholey MH, Chamorro HA, Rao G, Ford WB, Miller WH (1976) Bronchial artery embolization for massive hemoptysis. JAMA 236: 2501-2504.

11. Swanson KL, Johnson CM, Prakash UB, McKusick MA, Andrews JC, et al. (2002) Bronchial artery embolization: Experience with 54 cases. Chest 21 : 789-795.

12. Cremaschi P, Nascimbene C, Vitulo P, Catanese C, Rota L, et al. (1993) Therapeutic embolization of bronchial artery: a successful treatment in 209 cases of relapse hemoptysis. Angiology 44: 295-299.

13. Rabkin JE, Astafjev VI, Gothman LN, Grigorjev YG (1987) Transcatheter embolization in the management of pulmonary hemorrhage. Radiology 163: 361-365.

14. Mal H, Rullon I, Mellot F, Brugière O, Sleiman C, et al. (1999) Immediate and long-term results of bronchial artery embolization for life threatening hemoptysis.Chest115: 996-1001. 